\title{
Finite Memory-Length Linear Multiuser Detection for Asynchronous CDMA Communications
}

\author{
Markku J. Juntti, Student Member, IEEE, and Behnaam Aazhang, Senior Member, IEEE
}

\begin{abstract}
Decorrelating, linear, minimum mean-squared error (LMMSE), and noise-whitening multiuser detectors for code-division multiple-access systems (CDMA) are ideally infinite memory-length (referred to as IIR) detectors. To obtain practical detectors, which have low implementation complexity and are suitable for CDMA systems with time-variant system parameters (e.g., the number of users, the delays of users, and the signature waveforms), linear finite-memory-length (referred to as FIR) multiuser detectors are studied in this paper. They are obtained by truncating the IIR detectors or by finding optimal FIR detectors. The signature waveforms are not restricted to be timeinvariant (periodic over symbol interval). Thus, linear multiuser detection is generalized to systems with spreading sequences longer than the symbol interval. Conditions for the stability of the truncated detectors are discussed. Stable truncated detectors are shown to be near-far resistant if the received powers are upper bounded, and if the memory length is large enough (but finite). Numerical examples demonstrate that moderate memory lengths are sufficient to obtain the performance of the IIR detectors even with a severe near-far problem.
\end{abstract}

Index Terms - Code-division multiaccess, linear multiuser detector, spread-spectrum communication.

\section{INTRODUCTION}

$\mathbf{T}$ HE optimal multiuser detector [1] for code-division multiple-access (CDMA) systems has high computational complexity. For that reason, several suboptimal multiuser detectors have been proposed; see e.g., [2] and references therein. In the class of linear multiuser detectors, the decorrelating [3], [4], linear, minimum mean-squared error (LMMSE) [5], and the noise-whitening detectors ${ }^{1}$ [6] have received the most interest due to their good performance and simple mathematical formulation. The above detectors can be characterized as an inverse of some form of correlation matrix. In an ideal implementation, their memory length equals the number of users times the data packet length, which often can be assumed to approach infinity. Variations in

Paper approved by S. L. Miller, the Editor for Spread Spectrum of the IEEE Communications Society. Manuscript received March 19, 1996; revised October 14, 1996. This work was supported in part by the Academy of Finland, Graduate School in Electronics, Telecommunications, and Automation; Elektrobit Ltd.; the Finnish Air Force; Nokia Cellular Systems; Nokia Mobile Phones; and the Technology Development Centre of Finland.

M. J. Juntti was with the Department of Electrical and Computer Engineering, Rice University, Houston, TX 77251-1892 USA. He is now with the University of Telecommunication Laboratory, University of Oulu, FIN-90571 Oulu, Finland (e-mail: markku.juntti@ee.oulu.fi).

B. Aazhang is with the Department of Electrical and Computer Engineering, Rice University, Houston, TX 77251-1892 USA. (e-mail: aaz@ rice.edu).

Publisher Item Identifier S 0090-6778(97)03714-8.

${ }^{1} \mathrm{~A}$ noise-whitening detector is a linear detector per se; however, it often utilizes decision feedback or some tree-search algorithm. the number of users, in their signature waveforms (e.g., due to a handover in a cellular system), or in their delays change the correlations. In such a case, the multiuser detectors must be updated accordingly to match the new received signal. This is a computationally intensive operation if the memory length of the detectors is very large.

Several ways to obtain finite memory-length multiuser detectors have been proposed. The most natural way is to leave regular symbol intervals without transmission. This will result in finite blocks of transmitted symbols, and obviously, the detectors would then have finite memory length [7], [8]; in [7], such such an approach was called "isolation bit insertion." This, however, degrades the bandwidth efficiency and requires some form of synchronism between users. Other approaches to obtain finite-memory-length multiuser detectors include nonlinear subtraction of estimated multiple-access interference (MAI) ("edge correction") [9], and hard decision approximation of the decorrelator [5], which ends up in the decision-directed, nonlinear MAI canceler. The methods yield nonlinear detectors. The infinite memory length also has been one motivation to introduce adaptive, decentralized, oneshot multiuser detectors ${ }^{2}$ [10]-[16]. The drawbacks of these detectors are that they may require long adaptation times, and the adaptation must possibly be repeated frequently [17]. The training sequences required in most adaptive detectors degrade the bandwith efficiency, especially if the adaptation must occur frequently. Furthermore, the one-shot approach is inherently suboptimal, even in the class of linear detectors.

In this paper, we show that the infinite-memory-length detectors can be accurately approximated by detectors with finite and also relatively short memory length. In particular, we show that near-far resistance to a high degree can be obtained by moderate memory lengths. This result provides a mechanism to implement near-far resistant linear multiuser detectors in systems in which the number of users or their propagation delays change over time. A related problem has been discussed in [5] and in [18], but only the special cases of the LMMSE and noise-whitening detectors were considered. Furthermore, the emphasis in [5], [18] is on algorithm derivations; the stability and performance analysis of linear finite-memory-length detectors were not presented.

With only few exceptions [7], [8], [18], the multiuser detection has been considered for systems where the signature waveforms are periodic with period equaling the symbol

\footnotetext{
${ }^{2}$ Centralized multiuser detectors make a joint detection of the symbols of different users. Decentralized multiuser detectors (sometimes also called single-user detectors) demodulate a signal of one desired user only.
} 
interval. The signature waveforms in these systems are usually carefully designed to have good correlation properties [19, part 1, sect. 5.7]; such CDMA systems have been referred to as D-CDMA or deterministic CDMA systems [20] since the signature waveforms appear as periodic, deterministic signals. We also demonstrate in this paper that linear finite-memory-length multiuser detection can be generalized to CDMA systems where the signature waveforms vary from symbol interval to symbol interval. This allows one to use spreading sequences longer than symbol duration, for example. In these systems, the signature waveforms are usually part of a long noise-like pseudorandom sequence. Therefore, they have been referred to as R-CDMA or random CDMA [20] since the signature waveforms appear as random-like signals. Long spreading sequences are used, e.g., in the IS-95 standard for CDMA systems [21]. The generalization of linear multiuser detection to systems with time-variant signature waveforms provides new possibilities to improve the performance of systems with R-CDMA. A practical algorithm for implementation of finitememory-length linear detectors for systems with time-variant signature waveforms has been introduced in [22] and [23].

The outline of the paper is as follows. The CDMA system model and linear multiuser detectors are defined in Section II. The results of the stability analysis of finite-memorylength detectors are presented in Section III. The effects of the finite-memory length on the bit-error probability, the asymptotic multiuser efficiency, and the near-far resistance of the detectors are analyzed in Section IV. In Section V, the results are illustrated by numerical examples.

\section{PReliminaries}

\section{A. CDMA System Model}

The complex envelope of the received CDMA signal is assumed to be

$$
r(t)=\sum_{n=0}^{N_{b}-1} \sum_{k=1}^{K} b_{k}^{(n)} A_{k} s_{k}^{(n)}\left(t-n T-\tau_{k}\right)+z(t)
$$

where $N_{b}$ is the number of symbols in the data packet, $K$ is the number of users, $b_{k}^{(n)} \in \Xi$ is the data symbol of user $k$ at the interval $t \in[n T,(n+1) T), \Xi$ is the symbol alphabet, $A_{k}=\sqrt{2 E_{k}} e^{j \phi_{k}}$ is the received complex amplitude ${ }^{3}$ (assumed to be constant over the transmission), $E_{k}$ is the energy per symbol, $\phi_{k}$ is the carrier phase, $T$ is the symbol duration, $\tau_{k} \in[0, T)$ is the delay of the $k$ th signal, $z(t)$ is complex zero-mean additive white Gaussian noise process with twosided power spectral density $\sigma^{2}$, and $s_{k}^{(n)}(t)$ is the $k$ th user's signature waveform (assumed to be real for simplicity, but the analysis can be straightforwardly generalized to the complex case) with properties $s_{k}^{(n)}(t)=0$, if $t \notin[0, T)$, and $\int_{0}^{T}\left|s_{k}^{(n)}(t)\right|^{2} d t=1$. If the signature waveforms are periodic with period $T$, they will be called time invariant; otherwise, time variant. Constant envelope modulation (e.g., MPSK) is assumed, therefore, $|b|=1, \forall b \in \Xi$.

\footnotetext{
${ }^{3}$ The factor 2 in front of $E_{k}$ is included due to the complex envelope model; see, e.g., [24, Sect. 4-1].
}

The received signal will be processed in processing windows of length $N=2 P+1$, where $P$ is a positive integer and the window length $N$ is also called the detector memory length measured in symbol durations $T$. Note that we are not making any assumptions about the data packet length $N_{b}$. The symbol vector for time interval $t \in[n T,(n+1) T)$ is defined to be $\mathbf{b}^{(n)}=\left(b_{1}^{(n)}, b_{2}^{(n)}, \cdots, b_{K}^{(n)}\right)^{\top} \in \Xi^{K}$. A concatenation of received symbols over a processing window is denoted by ${ }^{4}$

$$
\boldsymbol{b}=\left(\mathbf{b}^{\top(n-P)}, \cdots, \mathbf{b}^{\top(n)}, \cdots, \mathbf{b}^{\top(n+P)}\right)^{\top} \in \Xi^{N K} .
$$

The sampled output of the $k$ th matched filter is $y_{k}^{(n)}=$ $\int_{n T+\tau_{k}}^{(n+1) T+\tau_{k}} r(t) s_{k}^{(n)}\left(t-n T-\tau_{k}\right) d t$. The sample vector for time interval $t \in[n T,(n+1) T)$ is defined as $\boldsymbol{y}^{(n)}=$ $\left(y_{1}^{(n)}, y_{2}^{(n)}, \cdots, y_{K}^{(n)}\right)^{\top} \in \mathbb{C}^{K}$, and their concatenation

$$
\boldsymbol{y}=\left(\mathbf{y}^{\top(n-P)}, \cdots, \mathbf{y}^{\top(n)}, \cdots, \mathbf{y}^{\top(n+P)}\right)^{\top} \in \mathbb{C}^{N K} .
$$

The former vector has expression [3]

$$
\begin{aligned}
\mathbf{y}^{(n)}= & \mathbf{R}^{(n)}(1) \mathbf{A} \mathbf{b}^{(n-1)}+\mathbf{R}^{(n)}(0) \mathbf{A} \mathbf{b}^{(n)} \\
& +\mathbf{R}^{(n)}(-1) \mathbf{A} \mathbf{b}^{(n+1)}+\mathbf{w}^{(n)}
\end{aligned}
$$

where $\mathbf{R}^{(n)}(i) \in[-1,1]^{K \times K}$ is a correlation matrix with $k l$ th element $R_{k l}^{(n)}(i)=\int_{-\infty}^{\infty} s_{k}^{(n)}\left(t-\tau_{k}\right) s_{l}^{(n-i)}\left(t+i T-\tau_{l}\right) d t, A=$ $\operatorname{diag}\left(A_{1}, \cdots, A_{K}\right) \in \mathbb{C}^{K \times K}$ is a diagonal matrix of received amplitudes, and $\boldsymbol{w}^{(n)} \in \mathbb{C}^{K}$ is the output vector due to noise. As in the case of time-invariant signature waveforms, it is easy to show that $\mathbf{R}^{(n)}(i)=\mathbf{0}, \quad \forall|i|>1$, and $\mathbf{R}^{(n)}(-1)=$ $\mathbf{R}^{T(n+1)}(1)$. The concatenation vector of the matched filter outputs has expressions

$$
\begin{aligned}
\boldsymbol{y} & =\mathcal{R} \mathcal{A} \boldsymbol{b}+\mathcal{R}_{e} \mathcal{A}_{e} \boldsymbol{b}_{e}+\boldsymbol{w} \\
& =\overline{\mathcal{R} \mathcal{A} \bar{b}}+\boldsymbol{w}
\end{aligned}
$$

where the vector $\boldsymbol{b}_{e}=\left(\mathbf{b}^{\top(n-P-1)}, \mathbf{b}^{\top(n+P+1)}\right)^{\top} \in \Xi^{2 K}$ includes the symbols outside the processing-window, $\overline{\boldsymbol{b}}=$ $\left(\mathbf{b}^{\top(n-P-1)}, \boldsymbol{b}^{\top}, \mathbf{b}^{\top(n+P+1)}\right)^{\top} \in \Xi^{(N+2) K}$,

$$
\begin{aligned}
\mathcal{A}= & \operatorname{diag}(\mathbf{A}, \cdots, \mathbf{A})^{\top} \in \mathbb{C}^{N K \times N K} \\
\mathcal{A}_{e}= & \operatorname{diag}(\mathbf{A}, \mathbf{A})^{\top} \in \mathbb{C}^{N K \times N K} \\
\overline{\mathcal{A}}= & \operatorname{diag}(\mathbf{A}, \mathcal{A}, \mathbf{A})^{\top} \in \mathbb{C}^{(N+2) K \times(N+2) K} \\
\mathcal{R}= & \left(\begin{array}{cccc}
\mathbf{R}^{(n-P)}(\mathbf{0}) & \mathbf{R}^{\top(n-P+1)}(1) & \cdots & \mathbf{0}_{K} \\
\mathbf{R}^{(n-P+1)}(1) & \mathbf{R}^{(n-P+1)}(\mathbf{0}) & \cdots & \mathbf{0}_{K} \\
\vdots & \vdots & & \vdots \\
\mathbf{0}_{K} & \mathbf{0}_{K} & \cdots & \mathbf{R}^{(n+P)}(0)
\end{array}\right) \\
& \in \mathbb{R}^{N K \times N K} \\
\mathcal{R}_{e}= & \left(\boldsymbol{\zeta}_{1}, \boldsymbol{\zeta}_{2}\right) \in \mathbb{R}^{N K \times 2 K}, \\
& \zeta_{1}=\left(\mathbf{R}^{\top(n-P-1)}(1), \mathbf{0}_{K}, \cdots, \mathbf{0}_{K}\right)^{\top} \in \mathbb{R}^{N K \times K} \\
& \zeta_{2}=\left(\mathbf{0}_{K}, \cdots, \mathbf{0}_{K}, \mathbf{R}^{(n+P+1)}(1)\right)^{\top} \in \mathbb{R}^{N K \times K}
\end{aligned}
$$

$\mathbf{0}_{K}$ is a $K \times K$ zero matrix, and $\overline{\mathcal{R}}=\left(\boldsymbol{\zeta}_{1}, \mathcal{R}, \boldsymbol{\zeta}_{2}\right) \in$ $\mathbb{R}^{N K \times(N+2) K}$. In (3), the first term is the response due to

${ }^{4}$ For notational convenience, the time index $n$ is left out from the definition of $\boldsymbol{b}$ and also from a few other expressions, when it is possible without confusion. Furthermore, a boldface, lower case, Roman (not italic) symbol (e.g., $\mathbf{b}^{(n)}$ ) denotes a vector of $K$ variables (e.g., $b_{k}^{(n)}$ ) over one symbol interval. A boldface, lower case, italic symbol (e.g., $\boldsymbol{b}$ ) denotes a vector of $N K$ variables concatenated over $N$ symbol intervals. 
symbols $\boldsymbol{b}$ inside the processing window, and the second is the response due to symbols $\boldsymbol{b}_{e}$ outside the processing window. The third term $\boldsymbol{w} \in$ comple $x^{N K}$ is the response due to noise, which is a zero-mean Gaussian random vector with covariance matrix $\sigma^{2} \mathcal{R}$. Expression (4) is obtained by writing the first two terms in (3) as one matrix-vector product. We assume that matrix $\mathcal{R}$ is positive definite, which is the case with probability one [3].

\section{B. Linear Multiuser Detectors}

Linear multiuser detectors process the matched filter output vector $\boldsymbol{y}$ by a linear operation. In other words, the detector output $\hat{\boldsymbol{h}}$ is $\hat{\boldsymbol{h}}=\boldsymbol{T}^{\top} \boldsymbol{y}$. If $N=N_{b}$, we have ideal detectors for packetized transmission. The decorrelating detector is $\mathcal{T}=\mathcal{R}^{-1}$ [3], and the noise-whitening detector is $\mathcal{T}=$ $\mathcal{L}^{-1}$, where $\mathcal{L}$ is the lower triangular Cholesky factor of $\mathcal{R}$ such that $\mathcal{R}=\mathcal{L}^{\top} \mathcal{L}$ [6]. ${ }^{5}$ If the information symbols $b_{k}^{(n)}$ are independent and uniformly distributed, the LMMSE detector is $\mathcal{T}=\left(\mathcal{R}+\sigma^{2} \mathcal{E}^{-1}\right)^{-1}$ [27], where $\mathcal{E}=\mathcal{A}^{\mathrm{H}} \mathcal{A}=$ $\operatorname{diag}(\mathbf{E}, \mathbf{E}, \cdots, \mathbf{E}) \in \mathbb{R}^{N K \times N K}$ and

$$
\mathbf{E}=\operatorname{diag}\left(2 E_{1}, 2 E_{2}, \cdots, 2 E_{K}\right) \in \mathbb{R}^{K \times K} .
$$

If the size of the data packet is very large, the ideal detectors described above may not be feasible. To obtain more practical detectors, we define a general finite-memory-length linear multiuser detector (referred to as an FIR detector for brevity) as ${ }^{6}$

$$
\begin{aligned}
\mathcal{D}= & (\mathbf{D}(P), \cdots, \mathbf{D}(1), \mathbf{D}(0), \mathbf{D}(-1), \cdots, \mathbf{D}(-P))^{\top} \\
& \in \mathbb{R}^{N K \times K}
\end{aligned}
$$

where the blocks $\mathrm{D}(i) \in \mathbb{R}^{K \times K}, i \in\{-P, \cdots, P\}$ define a partition of the detector $\mathcal{D}$. We define the infinite-memorylength linear multiuser detector (referred to as an IIR detector) corresponding to an FIR detector to be the limiting detector obtained by letting the memory length of the FIR detector approach infinity. In other words, the IIR detector is the limit of $\mathcal{D}$ as $N \rightarrow \infty$.

The linear multiuser FIR detector output $\hat{\mathbf{h}}^{(n)}=\mathcal{D}^{\top} \mathbf{y} \in$ $\mathbb{C}^{K}$ provides a decision statistic for the symbols $\boldsymbol{b}^{(n)}$. The output can be expressed as

$$
\hat{\mathbf{h}}^{(n)}=\mathcal{F}^{\top} \mathcal{A} \boldsymbol{b}+\boldsymbol{\mu}^{(n)}\left(\boldsymbol{b}_{e}\right)+\boldsymbol{\nu}^{(n)}=\overline{\mathcal{F}}^{\top} \overline{\mathcal{A}} \overline{\boldsymbol{b}}+\boldsymbol{\nu}^{(n)}
$$

where $\mathcal{F}=\mathcal{R D}$ and $\overline{\mathcal{F}}=\overline{\mathcal{R}}^{\top} \mathcal{D}$ are the convolutions of the multiuser channel impulse response $\mathcal{R}$ or $\overline{\mathcal{R}}$ and multiuser detector $\mathcal{D}$ :

$$
\begin{aligned}
\boldsymbol{\mu}^{(n)}\left(\boldsymbol{b}_{e}\right)= & \mathbf{D}(P) \mathbf{R}^{(n-P-1)}(1) \mathbf{A b}^{(n-P-1)} \\
& +\mathbf{D}(-P) \mathbf{R}^{\top(n+P+1)}(1) \mathbf{A} \mathbf{b}^{(n+P+1)}
\end{aligned}
$$

is the response of the symbols outside the processing window, i.e., the edge effect due to finite-detector memory length, and $\nu^{(n)}=\mathcal{D}^{\top} \boldsymbol{w}$ is a zero-mean Gaussian random vector

\footnotetext{
${ }^{5}$ The definition of Cholesky factorization used in this paper is an upper triangular matrix times a lower triangular matrix [25], [6] as opposed to the usual lower triangular times the upper triangular matrix [26].

${ }^{6}$ Note that the time index $n$ is again left out for notational convenience, although the detector $\mathcal{D}$ and the convolution matrix $\mathcal{F}$ depend on $n$.
}

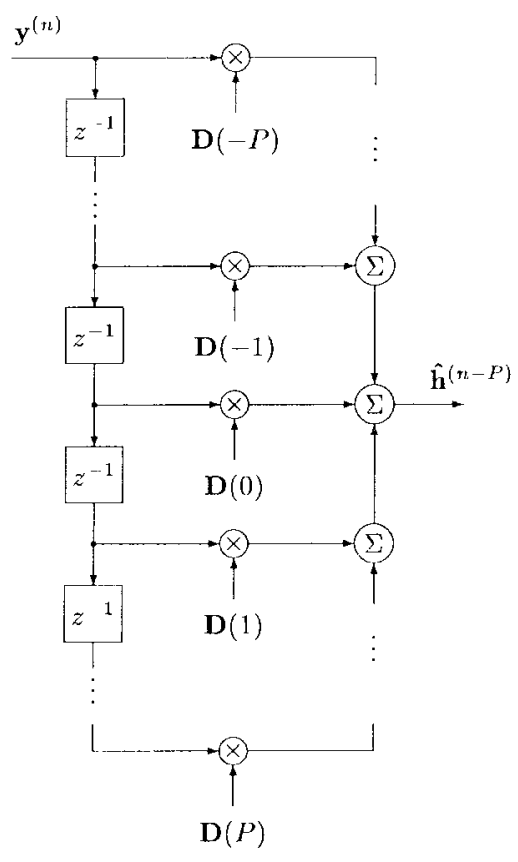

Fig. 1. FIR linear multiuser detector.

with covariance matrix $\sigma^{2} \mathcal{D}^{\top} \mathcal{R} \mathcal{D}$. In systems with timevariant signature waveforms, the above formulation should be interpreted as a snapshot of the time-variant detector on a particular symbol interval. Filtering interpretation of an arbitrary multichannel linear FIR detector is illustrated in Fig. 1.

To design FIR detectors, or in other words, to find in some sense good $N K \times K$ matrices $\mathcal{D}$, we first consider truncation of the IIR detectors, which was suggested in [3] for the decorrelating detector. A linear multiuser detector $\mathcal{D}_{d}$ satisfying

$$
\mathcal{R} \mathcal{D}_{d}=\mathcal{U}
$$

where

$\mathcal{U}=\left(\begin{array}{lllllll}\mathbf{0}_{K} & \cdots & \mathbf{0}_{K} & \mathbf{I}_{K} & \mathbf{0}_{K} & \cdots & \mathbf{0}_{K}\end{array}\right)^{\top} \in\{0,1\}^{N K \times K}$

will be called the truncated decorrelating detector. It is clear that $\mathcal{D}_{d}$ is the $N K \times K$ middle block column of the inverse of $\mathcal{R}$. A linear multiuser detector $\mathcal{D}_{m s}$ satisfying

$$
\left(\boldsymbol{R}+\sigma^{2} \mathcal{E}^{-1}\right) \mathcal{D}_{m s}=\mathcal{U}
$$

will be called the truncated LMMSE detector. ${ }^{7}$ A linear multiuser detector $\mathcal{D}_{n w}$ satisfying $\mathcal{L D}_{n w}=\mathcal{U}$ will be called the truncated noise-whitening detector.

An alternative to truncation is to optimize detectors based on the finite-processing window length model (4). To generalize the decorrelating detector we should find a zero-forcing detector, $\overline{\mathcal{D}}_{d}$ satisfying

$$
\overline{\mathcal{R}}^{\top} \overline{\mathcal{D}}_{d}=\mathcal{U}
$$

which does not have a unique solution. A unique detector can be found by selecting the pseudoinverse (i.e., Moore-Penrose

\footnotetext{
${ }^{7}$ Note that at high signal-to-noise ratios $\left(\sigma^{2} \rightarrow 0\right)$ or at high interference levels $\left(E_{k} \rightarrow \infty\right)$, the LMMSE detector approaches the decorrelating detector.
} 
generalized inverse), which yields the best least squares solution to (11). Since $\mathcal{R}$ is positive definite, $\overline{\mathcal{R}}$ has full rank with more columns than rows. Thus, the pseudoinverse solution defines the optimal FIR decorrelating detector

$$
\overline{\mathcal{D}}_{d}=\operatorname{mbc}\left\{\left(\overline{\mathcal{R \mathcal { R }}}^{\top}\right)^{-1} \overline{\mathcal{R}}\right\}
$$

where mbc denotes "middle block column of." It should be noted that the above detector is the optimal FIR decorrelator in the sense that it minimizes the least squares error in the solution of (11). However, there is no guarantee that the detector $\overline{\mathcal{D}}_{d}$ would yield lower bit-error probability than the truncated decorrelating detector $\mathcal{D}_{d}$. Since the optimal FIR detector forces the MAI due to edge symbols $\boldsymbol{b}_{e}$ to minimum, it cannot force the MAI anymore due to symbols $\boldsymbol{b}$ to zero; this tradeoff can introduce performance penalty in some cases.

The optimal FIR LMMSE detector is, by (4) and [27, sect. $12.5],{ }^{8}$

$$
\overline{\mathcal{D}}_{m s}=\operatorname{mbc}\left\{\mathcal{R}^{-1} \overline{\mathcal{R}}\left(\overline{\mathcal{R}}^{\top} \mathcal{R}^{-1} \overline{\mathcal{R}}+\sigma^{2} \overline{\mathcal{E}}^{-1}\right)^{-1}\right\}
$$

where $\overline{\mathcal{E}}=\operatorname{diag}(\boldsymbol{E}, \mathcal{E}, \boldsymbol{E}) \in \mathbb{R}^{(N+2) K \times(N+2) K}$. If all diagonal values of $\sigma^{2} \overline{\mathcal{E}}^{-1}$ are nonzero, matrix $\left(\overline{\mathcal{R}}^{\top} \mathcal{R}^{-1} \overline{\mathcal{R}}+\sigma^{2} \overline{\mathcal{E}}^{-1}\right)$ is nonsingular and (13) has a unique solution. If $\sigma^{2} \rightarrow 0$, there is no noise term in the model in (4), and the problem can be viewed to be deterministic and underdetermined. In that case, there is no LMMSE detector. Obviously, it is computationally simpler to update the truncated detectors than the optimal ones. What is more, computation of the truncated FIR detectors is numerically more stable in practical implementations. However, we choose to study both classes of FIR detectors for completeness.

It is clear that the use of FIR detectors instead of the IIR ones causes some performance loss. The performance analysis of the FIR detectors will be shown in Section IV. However, to be able to quantify the performance loss, we analyze the stability of linear multiuser detectors in the next section.

\section{Stability of Detectors}

In this section, we first discuss the conditions for the stability of the multiuser detectors. Although it proves to be impossible to find an easy test for the detector stability, the analysis gives insight into the problem. What is more, the analysis provides us with tools to derive two interesting results for stable detectors.

A multiuser detector $\mathcal{D}$ is defined to be stable if and only if the impulse response of the IIR detector is decaying, i.e., $\mathbf{D}(-P)$, and $\mathbf{D}(P) \rightarrow \mathbf{0}_{K}$, as $N \rightarrow \infty$ (or, equivalently, $P \rightarrow$ $\infty)$. The above definition is the standard stability definition of a digital IIR filter [28, pp. 81-82]. It is intuitive that if a multiuser detector is stable, the IIR detector can be truncated to an FIR detector with little performance degradation if the memory length $N$ is large enough. This can be predicted from (8), where the response of the symbols outside the processing window satisfies $\boldsymbol{\mu}^{(n)} \rightarrow \mathbf{0}$, as $N \rightarrow \infty$.

\footnotetext{
${ }^{8}$ The same result in a different form has been derived in [5]; the expression in (13) is more appropriate for further derivations in subsequent sections than the expression given in [5, eq. (4.3)].
}

For systems with time-invariant signature waveforms, it was shown in [3] that the truncated decorrelating detector is stable if and only if $^{9}$

$$
\operatorname{det}\left[\boldsymbol{R}^{\top}(1) e^{j \omega}+\boldsymbol{R}(0)+\boldsymbol{R}(1) e^{-j \omega}\right] \neq 0, \quad \forall \omega \in[0,2 \pi] .
$$

It is clear that (14) is hard to evaluate for all possible delay combinations. Therefore, the most practical solution is to compute numerical examples to determine whether or not a detector is stable. ${ }^{10}$ This is particularly true for systems with time-variant signature waveforms, as will be discussed below.

The condition in (14) was derived via a $z$-domain approach, which is not applicable in systems with time-variant signature waveforms. For that reason a time-domain analysis is needed. ${ }^{11}$ We first add the dimension symbol $N$ to $\mathcal{R}$ in (5) to yield $\boldsymbol{\mathcal { R }}_{N}$. To simplify the notation, we denote the nonzero blocks in $i$ th block column of $\mathcal{R}_{N}$ by $\mathbf{R}_{i}^{\top}(1), \mathbf{R}_{i}(1)$, and $\mathbf{R}_{i+1}(0) \cdot{ }^{11}$ Let the inverse of $\mathcal{R}_{N}$ be

$$
\begin{array}{r}
\mathcal{T}_{N}=\boldsymbol{R}_{N}^{-1}=\left(\begin{array}{cccc}
\mathbf{T}_{11}(N) & \mathbf{T}_{21}^{\top}(N) & \cdots & \mathbf{T}_{N, 1}^{\top}(N) \\
\mathbf{T}_{21}(N) & \mathbf{T}_{22}(N) & \cdots & \mathbf{T}_{N, 2}^{\top}(N) \\
\vdots & \vdots & & \vdots \\
\mathbf{T}_{N, 1}(N) & \mathbf{T}_{N, 2}(N) & \cdots & \mathbf{T}_{N, N}(N)
\end{array}\right) \\
\in \mathbb{R}^{N K \times N K} \\
\end{array}
$$

where each $\mathbf{T}_{i j}(N) \in \mathbb{R}^{K \times K}$. The dependence on $N$ is included in the argument since the blocks are different for different $N$. Note that $\mathbf{D}_{d}(-P)=\mathbf{T}_{N, P+1}(N)$. Thus, the stability of the truncated decorrelating detector is equivalent to $\mathbf{T}_{N, P+1}(N) \rightarrow \mathbf{0}_{K}$, as $N \rightarrow \infty$. The following recursive expressions (16) and (17), which are proved in the Appendix, provide us with the tools to study the stability of the detectors. For any $i, j \in\{1,2, \cdots, N-1\}$,

$$
\begin{aligned}
\mathbf{T}_{i, j}(N)= & \mathbf{T}_{i, j}(N-1)+\mathbf{T}_{N-1, i}^{\top}(N-1) \mathbf{R}_{N}^{\top}(1) \\
& \cdot \mathbf{T}_{N, N}(N) \mathbf{R}_{N}(1) \mathbf{T}_{N-1, j}(N-1) \\
\mathbf{T}_{N, j}(N)= & \boldsymbol{T}_{N, N}(N) \mathbf{R}_{N}(1) \mathbf{T}_{N-1, j}(N-1) .
\end{aligned}
$$

For any $1 \leq i<N$, we obtain by induction from (17) that

$$
\mathbf{T}_{N, i}(N)=\prod_{j=i+1}^{N}\left[\mathbf{T}_{j, j}(j) \mathbf{R}_{j}(1)\right] \mathbf{T}_{i, i}(i) .
$$

A sufficient condition for the stability of the detector, that is, for

$$
\mathbf{T}_{N, i}(N) \rightarrow \mathbf{0}_{K}, \quad \text { as } N \rightarrow \infty,
$$

is $\left|\lambda_{\max }\left[\mathbf{R}_{j}^{\top}(1) \mathbf{T}_{j, j}^{-2}(j) \mathbf{R}_{j}(1)\right]\right|<1, \forall j \in\{i+1, i+2, \cdots, N\}$ $\left[29\right.$, p. 69], where $\lambda_{\max }(\mathbf{A})$ denotes the eigenvalue of a matrix $\mathbf{A}$ with largest absolute value. The above condition, however, is often overly stringent. It was not satisfied in

\footnotetext{
${ }^{9}$ Time index $n$ is not needed in $R(i)$ since signature waveforms are time invariant.

${ }^{10}$ In the second column, for example, $\mathbf{R}_{2}^{\top}(1)=$ $\mathbf{R}^{\top(n-P+1)}(1), \mathbf{R}_{2}(0)=\mathbf{R}^{(n-P+1)}(0), \mathbf{R}_{3}(1)=\mathbf{R}^{(n-P+2)}(1)$, etc.

${ }^{11}$ The analysis we perform also applies to systems with time-invariant signature waveforms since the time-invariant case can be viewed as a special case of a system with time-variant signature waveforms.
} 
most of the numerical examples, but still the detectors were stable in all cases. In other words, it is very difficult to provide necessary conditions to the detector stability. Thus, the numerical examples are often the only practical way to verify the stability of the detectors.

For systems with time-invariant signature waveforms, it has been shown via the $z$-domain approach that the stability of the decorrelating detector implies the uniqueness of the limiting IIR detector [3]. The corresponding result for systems with time-variant signature waveforms is posed in the following proposition and is proved in the Appendix.

Proposition 1: If the truncated decorrelating, LMMSE, or noise-whitening detectors are stable, the limiting IIR detectors, obtained as $N \rightarrow \infty$, are unique.

The truncated detectors neglect the edge effect caused by the symbols outside the observation window, while the optimal FIR detectors take it into consideration. On the other hand, the stability of the detectors implies that the edge effect at the detector output approaches zero as $N$ is large. Thus, it is expected that the truncated and the optimal FIR detectors should approach the same limiting IIR detector if they are stable. This is indeed the case under mild conditions, as stated below and proved in the Appendix.

Proposition 2: Assume that the received energies $E_{k}$ and noise power spectral density $\sigma^{2}$ satisfy $0<\left(E_{k} / \sigma^{2}\right)<\infty, \forall k \in\{1,2, \cdots, K\}$. Assume also that the decorrelating and LMMSE detectors are stable. Then both the truncated LMMSE detector $\mathcal{D}_{m s}$ (10) and the optimal FIR LMMSE detector $\overline{\mathcal{D}}_{m s}$ (13) converge to the same IIR LMMSE detector as the detector memory length $N$ approaches infinity, i.e.,

$$
\lim _{N \rightarrow \infty} \overline{\mathcal{D}}_{m s}=\lim _{N \rightarrow \infty} \mathcal{D}_{m s}
$$

The corresponding result for the decorrelating detectors, as in 20, does not have as simple a formulation. However, both the truncated decorrelating detector $\mathcal{D}_{d}$ (9) and the optimal FIR decorrelating detector $\overline{\mathcal{D}}_{d}$ (12) converge to the same IIR decorrelating detector as the processing-window length $N$ approaches infinity, i.e.,

$$
\lim _{N \rightarrow \infty} \overline{\mathcal{D}}_{d}=\lim _{N \rightarrow \infty} \mathcal{D}_{d}
$$

under mild conditions. The conditions are discussed at the end of the Appendix.

It was noted in Section II-B that the truncated detectors are easier to compute than the optimal FIR detectors. Moreover, the above results justify the use of the truncated detectors with large enough memory length.

\section{Performance Analysis}

In the performance analysis, we assume BPSK data modulation, and that the carrier phases $\phi_{k}=0$. However, the extension to more general cases is straightforward. The delays are assumed to be fixed. The $k$ th users' average bit-error probability of a linear FIR detector is obtained by averaging over all possible interfering symbol combinations [30]. By (7), it can be expressed in the forms

$$
\begin{aligned}
\bar{P}_{k}= & \frac{1}{2^{(N+2) K-1}} \sum_{\substack{\overline{\boldsymbol{b}} \in\{-1,1\}^{(N+2) K} \\
b_{k}^{(n)}=0}} \\
& \cdot Q\left(\frac{\sqrt{2 E_{k}}(\mathcal{F})_{(P+1) K+k, k}-\bar{f}_{k}^{\top} \mathcal{A} \boldsymbol{b}}{\sqrt{\sigma^{2}\left[\mathcal{D}^{\top} \mathcal{R} \mathcal{D}\right]_{k k}}}\right) \\
= & \frac{1}{2^{(N+2) K-1}} \sum_{\substack{\overline{\boldsymbol{b}} \in\{-1,1)^{(N+2) K} \\
b_{k}^{(n)}=0}} \\
& \cdot Q\left(\frac{\sqrt{2 E_{k}}(\mathcal{F})_{(P+1) K+k, k}-\boldsymbol{f}_{k}^{\top} \mathcal{A} \boldsymbol{b}-\mu_{k}^{(n)}\left(\boldsymbol{b}_{e}\right)}{\sqrt{\sigma^{2}\left[\mathcal{D}^{\top} \mathcal{R} \mathcal{D}\right]_{k k}}}\right)
\end{aligned}
$$

where $Q(x)=(1 / \sqrt{2 \pi}) \int_{x}^{\infty} e^{-t^{2} / 2} d t$, and $\boldsymbol{f}_{k}$ and $\overline{\boldsymbol{f}}_{k}$ are the $k$ th columns of $\mathcal{F}$ and $\overline{\mathcal{F}}$ defined in (7), respectively. The term $\sqrt{2 E_{k}}(\mathcal{F})_{(P+1) K+k, k}$ is the desired signal component, $\overline{\boldsymbol{f}}_{k}^{\top} \mathcal{A} \boldsymbol{b}=\boldsymbol{f}_{k}^{\top} \mathcal{A} \boldsymbol{b}+\mu_{k}^{(n)}\left(\boldsymbol{b}_{e}\right)$ is the remaining MAI, and $\sigma^{2}\left[\mathcal{D}^{\top} \mathcal{R} \mathcal{D}\right]_{k k}$ is the Gaussian noise variance at the detector output. The expression for error probability of an IIR detector is as in (23) with $\mu_{k}^{(n)}\left(\boldsymbol{b}_{e}\right)=0$. It is easy to see from (8) and (23) that in the case of a stable detector, the effect of symbols $\mathbf{b}^{(n-P-1)}$ and $\mathbf{b}^{(n+P+1)}$ can be made arbitrarily small by selecting large enough memory length $N$. In the case of the decorrelating detector, this becomes even more clear. Since, by (9), we have $(\mathcal{F})_{(P+1) K+k, k}=1, \boldsymbol{f}_{k}=\mathbf{0}$ (except $\left.\left(\boldsymbol{f}_{k}\right)_{(P+1) K+k}=1\right)$, and $\left[\mathcal{D}^{\top} \mathcal{R} \mathcal{D}\right]_{k k}=\left[\boldsymbol{D}_{d}(0)\right]_{k k}$, then the error probability of the truncated decorrelator simplifies from (23) to

$$
\bar{P}_{d, k}=\frac{1}{2^{2 K}} \sum_{\mathbf{b}_{e} \in\{-1,1\}^{2 K}} \mathrm{Q}\left(\frac{\sqrt{2 E_{k}}-\mu_{d, k}^{(n)}\left(\mathbf{b}_{e}\right)}{\sqrt{\sigma^{2}\left[\mathbf{D}_{d}(0)\right]_{k k}}}\right) .
$$

If $N$ is large enough, $\left[\mathbf{D}_{d}(0)\right]_{k k}$ approaches the value of the IIR detector by Proposition 1 , and $\mu_{d, k}^{(n)}$ approaches zero if the decorrelator is stable. Thus, the stable decorrelator approaches the performance of the IIR detector with large enough memory length $N$.

In the following, the asymptotic multiuser efficiency (AME) and the near-far resistance (NFR) of linear FIR detectors will be analyzed. The AME of user $k$ is defined as [3]

$$
\eta_{k}=\sup \left\{0 \leq \varrho \leq 1: \lim _{\sigma^{2} \rightarrow 0} \frac{\bar{P}_{k}}{\mathrm{Q}\left(\sqrt{\frac{\varrho 2 E_{k}}{\sigma^{2}}}\right)}<\infty\right\} .
$$

The near-far resistance has been defined by $\bar{\eta}_{k}=$ $\inf _{E_{l} \geq 0, l \neq k} \eta_{k}$. The detector is said to be near-far resistant if $\bar{\eta}_{k}>0$. With large argument values, we can approximate $Q(x) \approx\left(\exp \left(-x^{2} / 2\right) / 2 \pi\right)$. At high signal-to-noise ratios, the worst case symbol combination dominates the value of the sum in the numerator of (22) or (23) [3]. Thus, using (23), 
the AME of an arbitrary linear FIR multiuser detector is

$$
\begin{aligned}
& \eta_{k}=\frac{1}{2 E_{k}} \max ^{2}\left\{0, \min _{\substack{\overline{\boldsymbol{b}} \in\{-1,1\}^{(N+2) K} \\
b_{k}(n)=0}}\right. \\
& \left.\cdot \frac{\sqrt{2 E_{k}}(\mathcal{F})_{(P+1) K+k, k}-f_{k}^{\top} \mathcal{A b}-\mu_{k}^{(n)}\left(\mathbf{b}_{e}\right)}{\sqrt{\left[\mathcal{D}^{\top} \mathcal{R} \mathcal{D}\right]_{k k}}}\right\} .
\end{aligned}
$$

The minimum above is obtained by the worst possible interfering symbol combination, i.e., with symbols $(\overline{\boldsymbol{b}})_{i}=$ $\operatorname{sgn}\left[\left(\bar{f}_{k}\right)_{i}\right], \forall i \in\{1,2, \cdots,(P+1) K+k-1,(P+1) K+$ $k+1, \cdots, N K\}$.

After evaluating the square in (25), the AME for the truncated decorrelating detector becomes

$$
\eta_{d, k}= \begin{cases}0, & \text { if } \mu_{d, k}^{\max } \geq \sqrt{2 E_{k}} \\ \frac{1-\rho_{d, k}}{D_{k k}(0)}, & \text { if } \mu_{d, k}^{\max }<\sqrt{2 E_{k}}\end{cases}
$$

where $\rho_{d, k}=\left(2 \sqrt{2 E_{k}} \mu_{d, k}^{\max }-\left(\mu_{d, k}^{\max }\right)^{2} / 2 E_{k}\right)$ describes the degradation due to edge effect, and

$$
\begin{aligned}
\mu_{d, k}^{\max }= & \max _{\boldsymbol{b}_{e} \in\{-1,1\}^{2 K}} \mu_{d, k}^{(n)}\left(\boldsymbol{b}_{e}\right) \\
= & \sum_{l \neq k}\left\{\left|\left[\mathbf{D}_{d}(P) \mathbf{R}(1)\right]_{k l}\right|\right. \\
& \left.+\left|\left[\mathbf{D}_{d}(-P) \mathbf{R}^{\top}(1)\right]_{k l}\right|\right\} \sqrt{2 E_{l}}
\end{aligned}
$$

is the maximum absolute value of $\mu_{d, k}^{(n)}$. The AME of the IIR decorrelator is as in (26) with $\rho_{d, k}=0$ since the edge effect has reduced to zero.

It can be verified from (26) that $\eta_{d, k}>0$ if and only if $\mu_{d, k}^{\max }<\sqrt{2 E_{k}}$. In other words, the truncated decorrelating detector has positive AME if and only if the maximum value of the remaining MAI component at the detector output is smaller than the desired users' amplitude. If any interfering amplitude approaches infinity, $\mu_{d, k}^{\max }$ at the output of an FIR detector also approaches infinity. Thus, it is clear that the FIR detectors cannot be near-far resistant in a strict sense. For that reason, we define power-limited near-far resistance by

$$
\bar{\eta}_{k}=\inf _{0 \leq E_{l} \leq E_{\max }, l \neq k} \eta_{k}
$$

where $E_{\max }$ is finite. In wireless communication systems, for example, $E_{\max }$ is determined by the accuracy of the power control of the CDMA system. If an FIR detector is stable, $\mu_{k}^{\max }$ can be made arbitrarily small by selecting $N$ large enough for any $E_{\max }$. This implies that the truncated decorrelating detector (and also LMMSE and data-aided ${ }^{12}$ noise-whitening detectors) with large enough (but finite) memory length can be made near-far resistant given an arbitrarily large (but finite) upper bound for the received powers of the interfering users. By (21), we note that, with $N$ large enough, the above discussion applies to the optimal FIR decorrelator as well.

\footnotetext{
${ }^{12}$ Data-aided (DA) stands for ideal decision feedback [6].
}

From (25), it is seen that the power-limited NFR for the FIR detectors can be computed by substituting $E_{l}=E_{\max } \forall l \neq$ $k$. Thus, the power-limited NFR is solely a function of the ratio $E_{\max } / E_{k}$. Let $E_{\min }$ be the minimum received energy a user needs to have to be served by the CDMA system. The worst case power-limited near-far resistance $\bar{\eta}_{k}$. can be computed by substituting $E_{k}=E_{\min }$ and $E_{l}=$ $E_{\max } \forall l \neq k$ in (25). In practice, $E_{\max }, E_{\min }$, and $N$ are design parameters of the CDMA system, and a tradeoff between them must be considered. The larger $N$, the more complicated the implementation of the detector is. On the other hand, large $N$ poses milder requirements for the power control of the system. In a DSP implementation, large $N$ introduces more roundoff errors and implementation noise so that, in practice, there is a finite optimal value for $N$ given the ratio $E_{\max } / E_{\min }$ and the implementation constraints (filter structure, floating-point number word length, etc.).

\section{NUMERICAL EXAMPLES}

The stability of the detectors was studied by numerical examples. Direct-sequence spread-spectrum waveforms and BPSK data and spreading modulation with coherent detection were considered. The number of users was 33 with spreading gain of 31 , i.e., the chip duration $T_{c}=T / 31$. The error probabilities were estimated for low signal-to-noise ratio by (22). Data-aided (DA) detection was assumed for the noisewhitening detector, which may not be practical. However, the effect of finite memory length can be well illustrated by examples assuming data-aided detection. The performance at high signal-to-noise ratios is evaluated by computing AME's using expression (25). The results are represented as a function of the parameter $P$. All of the interfering users were assumed to have the same energy, which is denoted by $E_{\max }$ in the figures; correspondingly, the energy of the desired user is denoted by $E_{\text {min }}$. The performance of the ideal IIR detector was estimated with the assumption that the edge symbols are zero, and the detector has large enough block size $(P=8$ proved to be sufficient).

A length-31 Gold code family was used in a system with time-invariant signature waveforms; the power-limited near-far resistances and error probabilities are depicted in Figs. 2 and 3. A random code family of length 6200 was used in a system with time-variant signature waveforms so that the results were averaged over $6200 / 31=200$ symbols; the power-limited near-far resistances and error probabilities are depicted in Figs. 4 and 5, where only the truncated decorrelating and the LMMSE detectors are considered since the analysis of the optimal FIR and noise-whitening detectors would be computationally intensive. For clarity, the bit error probabilities of the ideal LMMSE detector have not been plotted for the cases $E_{\min } / E_{\max }=-10 \mathrm{~dB}$ and $E_{\min } / E_{\max }=-20 \mathrm{~dB}$ in Figs. 3 and 5 since they are very close to the bit-error probability of the decorrelating detector.

It can be seen from Fig. 2 that the asymptotic loss in signal-to-noise ratio converges relatively fast. With $P=6$, the performance is the same as with an ideal IIR detector, even in the case $E_{\min } / E_{\max }=-20 \mathrm{~dB}$. With perfect power 


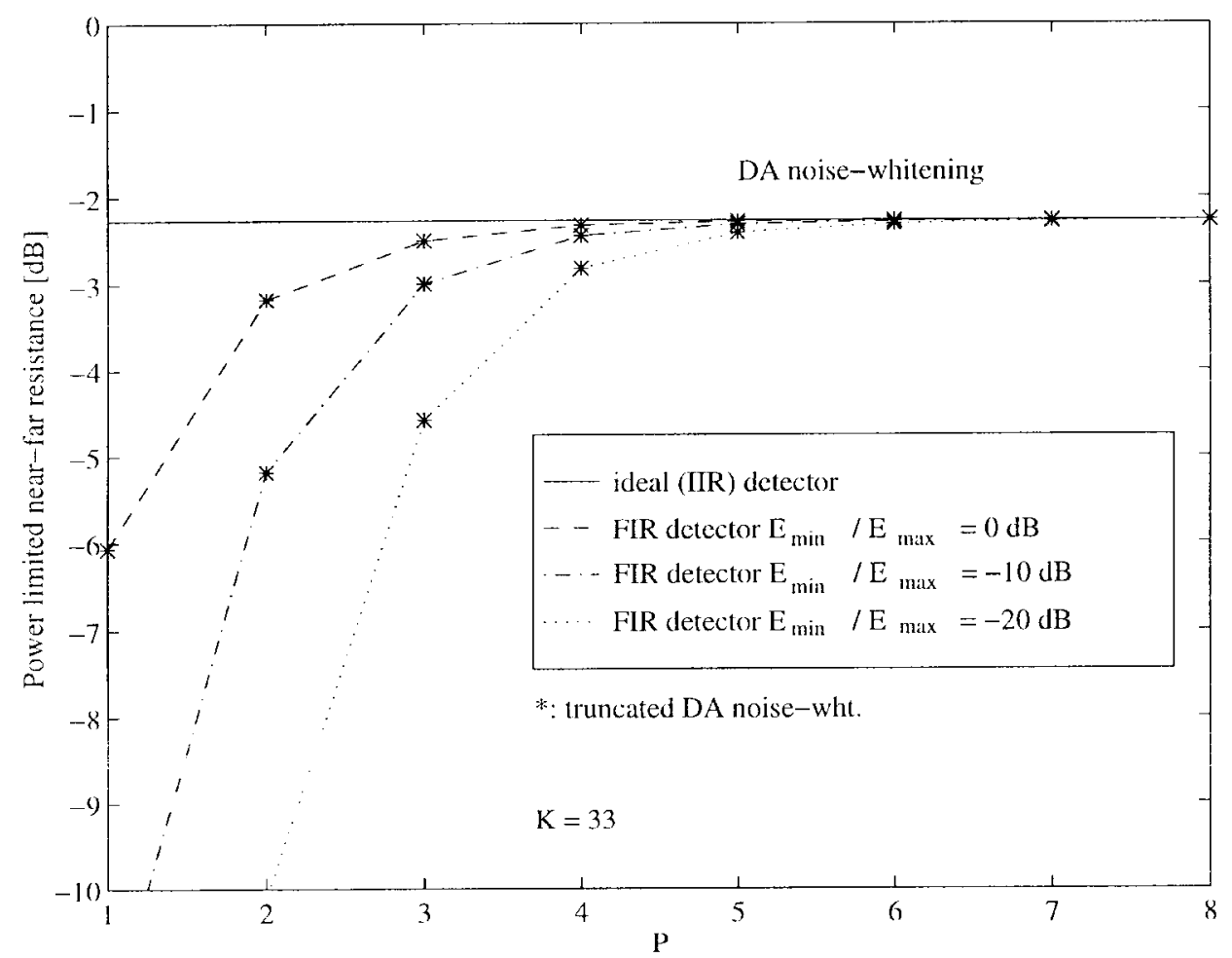

(a)

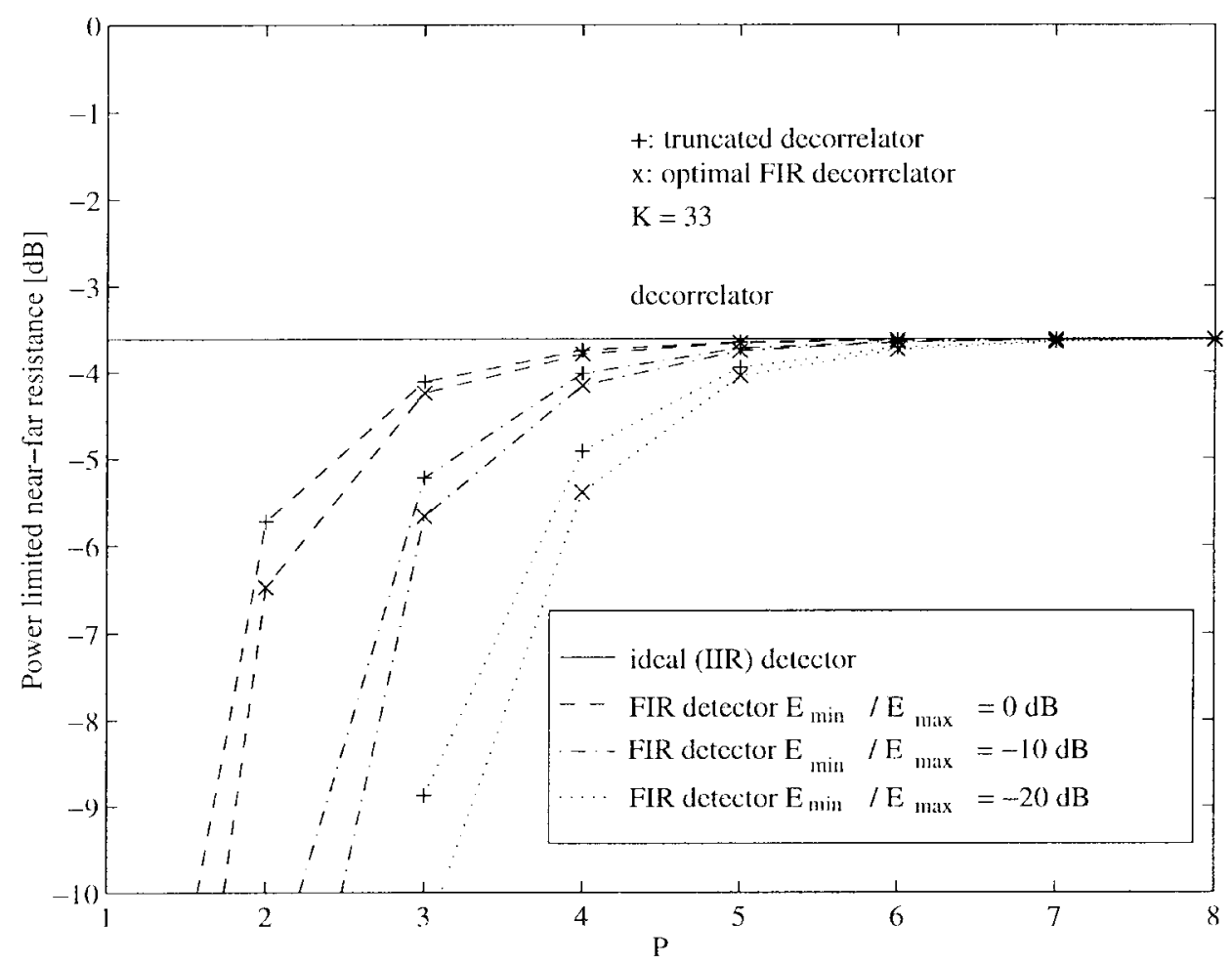

(b)

Fig. 2. Power-limited near-far resistances $[\mathrm{dB}]$ as a function of parameter $P$ with time-invariant signature waveforms. (a) DA truncated noise-whitening detector. (b) Truncated and optimal FIR decorrelating detector.

control $\left(E_{\min } / E_{\max }=0 \mathrm{~dB}\right)$, value $P=4$ is required. We also can see that a $10 \mathrm{~dB}$ increase in the MAI level implies that the value of $P$ must be roughly incremented by one to maintain the same performance. In other words, loosening the power-control requirements significantly calls for only a very minor increase in the required detector memory length. We see from Fig. 3 that, at lower signal-to-noise ratios, the value $P=4$ yields the same performance as the ideal 
IIR detector in all cases. We see from Figs. 4 and 5 that similar conclusions can be drawn for a system with timevariant signature waveforms. However, the performance of the ideal IIR detector is slightly better with the time-invariant than with time-variant signature waveforms; this is understandable due to the small cross correlations of Gold codes. On the other hand, a system with time-variant signature waveforms requires slightly smaller FIR detector memory lengths than the system with time-invariant signature waveforms, particularly at high signal-to-noise ratios. In [18], a similar behavior was observed and discussed for the noise-whitening detector. An intuitive explanation can be seen from (18). In systems with time-variant signature waveforms, the elements in the matrix $\mathbf{R}_{j}$ (1) are (at least approximately) random variables with mean zero and are independent for different values of $j$; thus, the elements of the matrix product $\mathbf{T}_{j, j}(j) \mathbf{R}_{j}(1)$ also have zero mean. In systems with time-invariant signature waveforms, on the other hand, the matrices are $\mathbf{R}_{j}(1)$, the same for different values of $j$, and there is "less randomness" in the elements of $\mathbf{T}_{j, j}(j) \mathbf{R}_{j}(1)$. Therefore, time-variant signature waveforms introduce more averaging out into the product in (18), and result in faster convergence of the detector to a zero matrix as the detector memory length $N \rightarrow \infty$.

From Figs. 3 and 5, it is seen that the optimal FIR detectors perform slightly better at low signal-to-noise ratios than truncated ones with small values of $P$. However, with moderate values of $P$, both are equivalent to the ideal IIR detectors, as is expected by Proposition 2. From Figs. 2 and 4 , we see that at high signal-to-noise ratios, on the other hand, the truncated decorrelating detector slightly outperforms the optimal FIR decorrelator. The reason can be understood from the expressions for AME. Although the contribution due to the symbols outside the processing window $\left(\mu_{k}^{(n)}\left(\boldsymbol{b}_{e}\right)\right)$ for the optimal FIR decorrelating detector in (25) is smaller than for the truncated FIR decorrelating detector in (26), the MAI due to other symbols $\boldsymbol{f}_{k}^{\top} \mathcal{A} \boldsymbol{b}$ in (25) is larger. Furthermore, the desired signal's energy $\sqrt{2 E_{k}}(\mathcal{F})_{(P+1) K+k, k}$ may be lower, and the enhanced additive white Gaussian noise $\sqrt{\left[\mathcal{D}^{\top} \mathcal{R D}\right]_{k k}}$ in (25) may be larger than the corresponding quantities in (26), yielding lower asymptotic multiuser efficiency. Thus, the optimal FIR detectors do not yield any universal performance improvement in comparison to the truncated detectors.

The numerical examples show that moderate memory lengths (roughly $N \leq 13$ ) give performance close to ideal IIR detectors in the cases studied. Even under a severe near-far problem $\left(E_{\min } / E_{\max }=-20 \mathrm{~dB}\right)$, we obtain the optimal near-far resistance with detector memory length $N=13$. The use of FIR detectors loosens the required accuracy of the power control significantly with very moderate detector memory lengths.

\section{CONCLUSIONS}

Linear multiuser detectors in asynchronous multiuser systems, whose signature waveforms are allowed to be time invariant or time variant, have been discussed. Two classes of linear FIR multiuser detectors, namely, the truncated and the optimal FIR detectors, were defined. The detectors were shown to be stable under relatively mild conditions. The stability was shown to imply asymptotic uniqueness of the limiting IIR detector. The truncated and the optimal FIR detectors asymptotically approach the same IIR detector under mild conditions. The performance of the finite memory-length detectors was analyzed. It was shown that the truncated decorrelating, LMMSE, and data-aided noise-whitening detectors can be made near-far resistant under a given ratio between maximum and minimum received power of users by selecting an appropriate memory length. Numerical examples demonstrate the fact that moderate memory lengths of either truncated or optimal FIR detectors are sufficient to gain the performance of the ideal IIR detectors, even under a severe near-far problem. If the memory lengths are short, the optimal FIR detectors outperform the truncated ones at low signalto-noise ratios. However, at high signal-to-noise ratios, the truncated detectors have better performance. The required memory lengths tend to be smaller with time-variant than with time-invariant signature waveforms.

The use of FIR detectors instead of the IIR detectors makes linear multiuser detection possible in CDMA systems in which the number of users, their propagation delays, or the signature waveforms change over time; an example of the time-variant signature waveforms is a CDMA system using spreading sequences longer than one symbol interval (an R-CDMA system). The truncated FIR detectors are easier to update to the changes in a communication system than the optimal FIR detectors. Because the optimal FIR detectors do not yield any universal performance improvement, the truncated detectors with appropriate memory length are clearly the detectors of choice in practice. The required memory length depends on other system parameters, especially on the ratio of maximum and minimum received powers.

APPENDIX

\section{STABILITY ANALYSIS}

Proof of Equalities (16) and (17): We define partitions

$$
\boldsymbol{R}_{N}=\left(\begin{array}{ll}
\boldsymbol{R}_{N-1} & \boldsymbol{\gamma}_{N-1} \\
\boldsymbol{\gamma}_{N-1}^{\top} & \boldsymbol{R}_{N}(0)
\end{array}\right) \in \mathbb{R}^{N K \times N K}
$$

where $\mathcal{R}_{N-1} \in \mathbb{R}^{(N-1) K \times(N-1) K}$

$$
\boldsymbol{\gamma}_{N-1}=\left(\mathbf{0}_{K} \cdots \mathbf{0}_{K} \boldsymbol{R}_{N}(1)\right)^{\top} \in \mathbb{R}^{(N-1) K \times K}
$$

and

$$
\mathcal{T}_{N}=\boldsymbol{R}_{N}^{-1}=\left(\begin{array}{cc}
\mathcal{C}_{N-1} & \boldsymbol{\alpha}_{N-1} \\
\boldsymbol{\alpha}_{N-1}^{\top} & T_{N, N}(N)
\end{array}\right) \in \mathbb{R}^{N K \times N K}
$$

where $\boldsymbol{C}_{N-1} \in \mathbb{R}^{(N-1) K \times(N-1) K}$ and $\boldsymbol{\alpha}_{N-1} \in \mathbb{R}^{(N-1) K \times K}$.

By applying the matrix inversion formulas [27, pp. 571-572], shown at the bottom of the page, and the fact that $\mathcal{R}_{N}$ and $\mathcal{T}_{N}$ are symmetric, we obtain the recursion formulas:

$$
\begin{aligned}
\mathcal{C}_{N-1} & =\mathcal{T}_{N-1}+\mathcal{T}_{N-1} \boldsymbol{\gamma}_{N-1} \boldsymbol{T}_{N, N}(N) \boldsymbol{\gamma}_{N-1}^{\top} \mathcal{T}_{N-1} \\
\boldsymbol{\alpha}_{N-1}^{\top} & =-\boldsymbol{T}_{N, N}(N) \boldsymbol{\gamma}_{N-1}^{\top} \mathcal{T}_{N-1} .
\end{aligned}
$$

Now, (16) and (17) follow by the definitions of $\mathcal{T}_{N-1}, \gamma_{N-1}$, and $\boldsymbol{\alpha}_{N-1}$. 


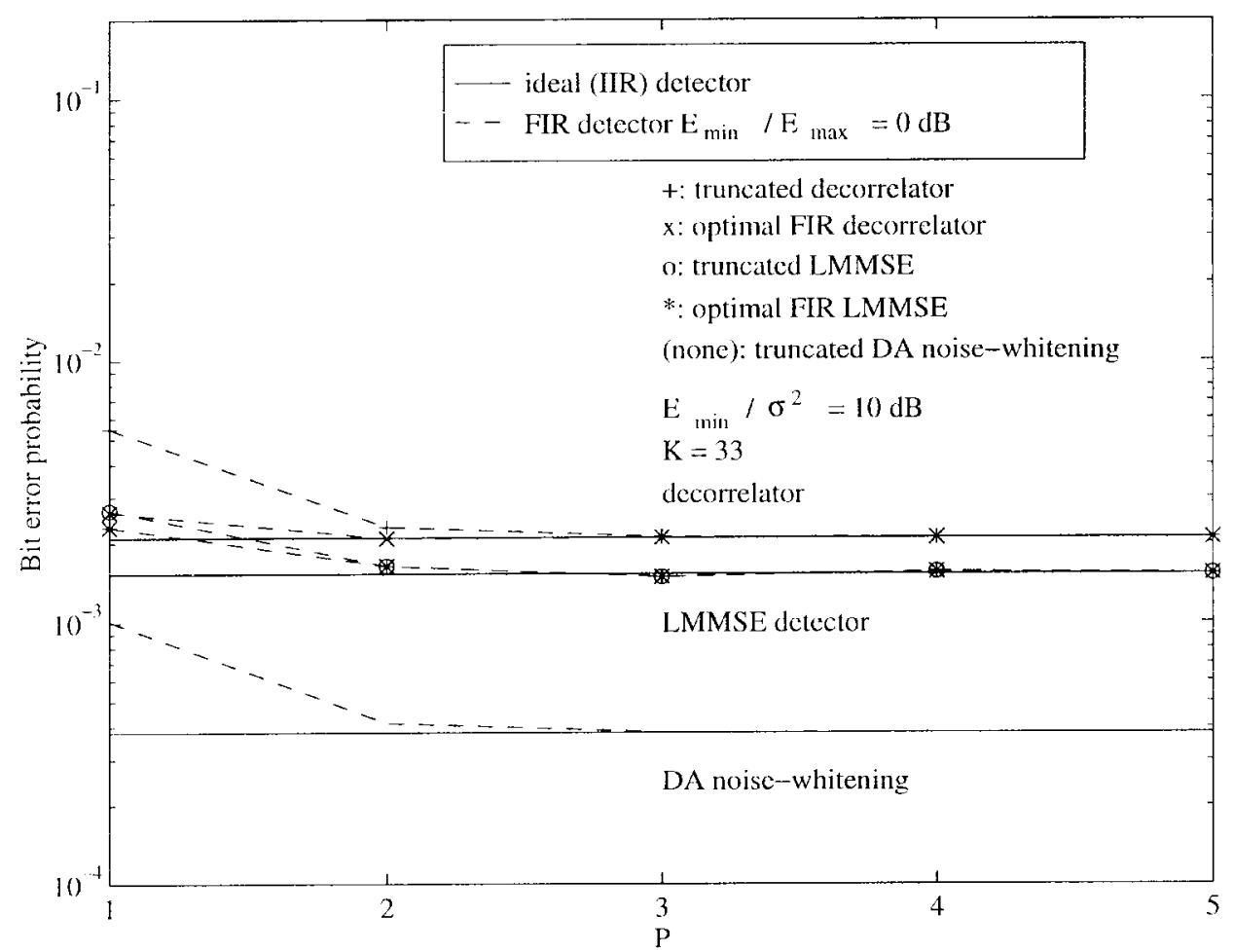

(a)

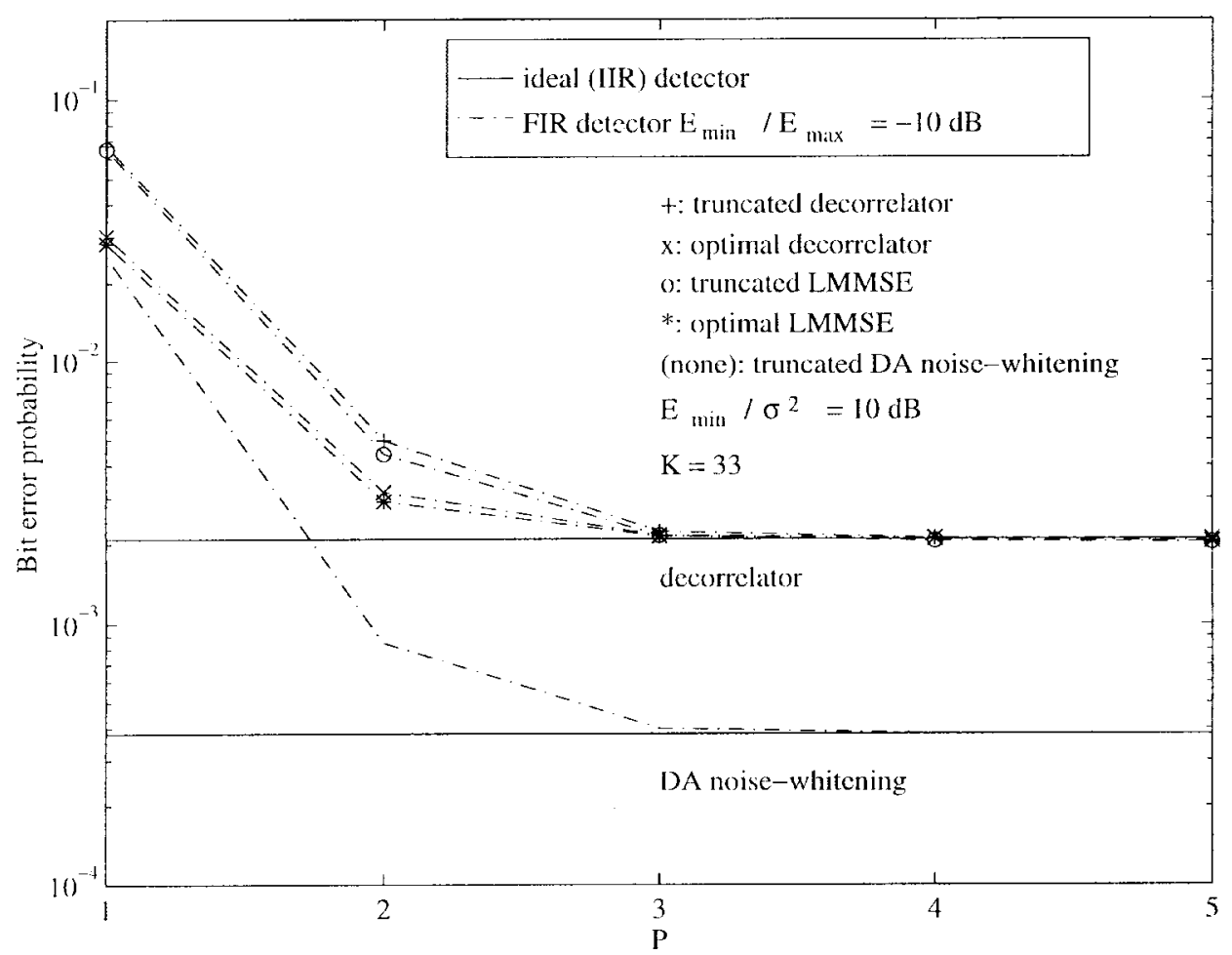

(b)

Fig. 3. Probabilities of bit error as a function of parameter $P$ with time-invariant waveforms. (a) Equal received energies $E_{\min } / E_{\max }=0 \mathrm{~dB}$. (b) Near-far problem $E_{\min } / E_{\max }=-10 \mathrm{~dB}$.

Proof of Proposition 1: Assume that the decorrelating detector is stable, and assume that integer $j$ is such that $N \rightarrow \infty$ implies $(N-j) \rightarrow \infty .{ }^{13}$ Then, we have by the stability of the

${ }^{13}$ The condition states that $j$ is such that its distance to $N$ approaches infinity as $N$ approaches infinity. The condition is satisfied, e.g., if $N=c j$, decorrelating detector, $\mathbf{T}_{N-1, j}(N-1) \rightarrow \mathbf{0}_{K}$ as $N \rightarrow \infty$. Assume that both $i$ and $j$ are such that $N \rightarrow \infty$ implies $(N-$ $i) \rightarrow \infty$ and $(N-j) \rightarrow \infty$. Then the increment part in (16)

where $c$ is an arbitrary constant. For example, since $N=2 P+1$, it follows that $N \rightarrow \infty \Rightarrow P \rightarrow \infty$. 


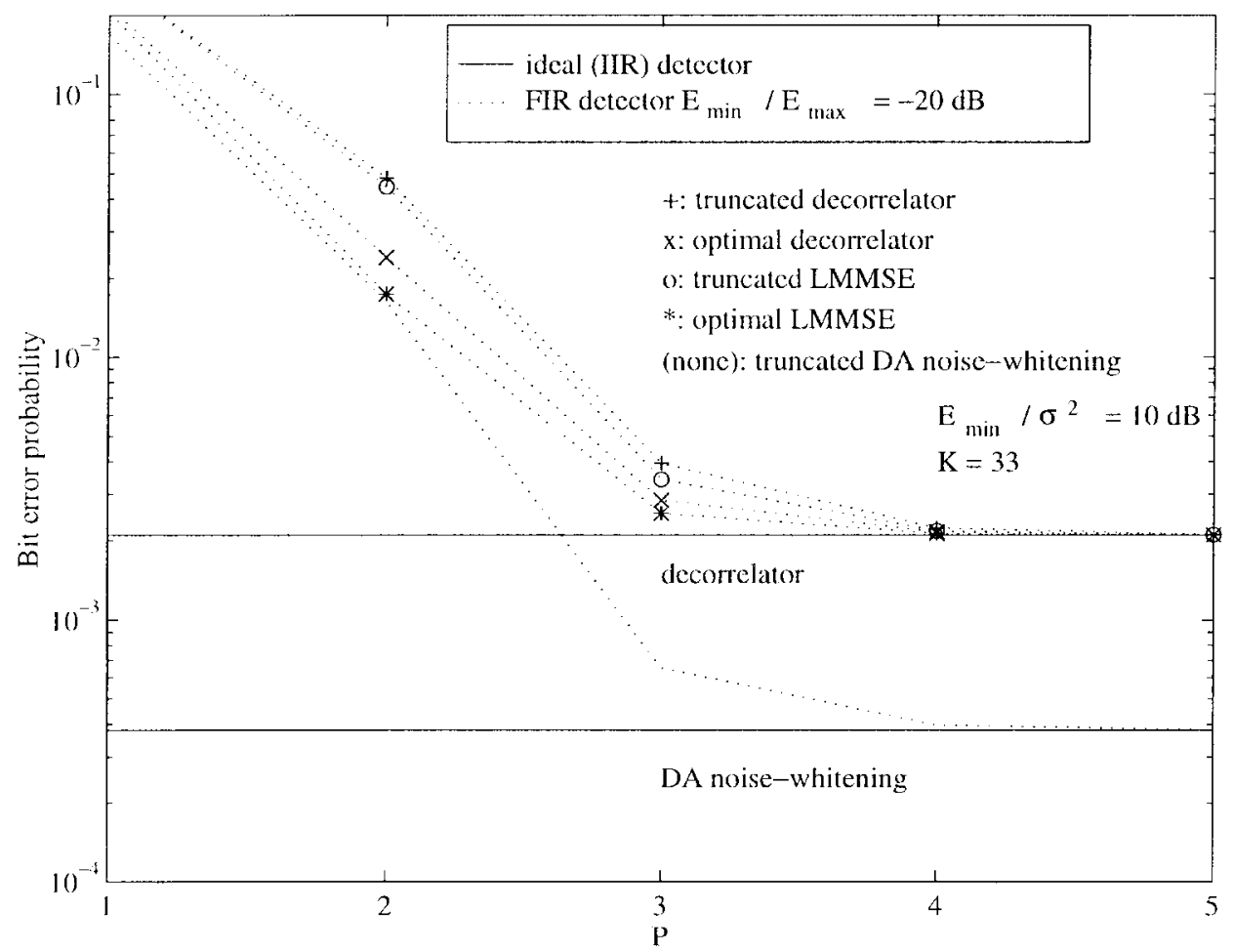

(c)

Fig. 3. (Continued.) Probabilities of bit error as a function of parameter $P$ with time-invariant signature waveforms. (c) Near-far problem $E_{\min } / E_{\max }=-20 \mathrm{~dB}$.

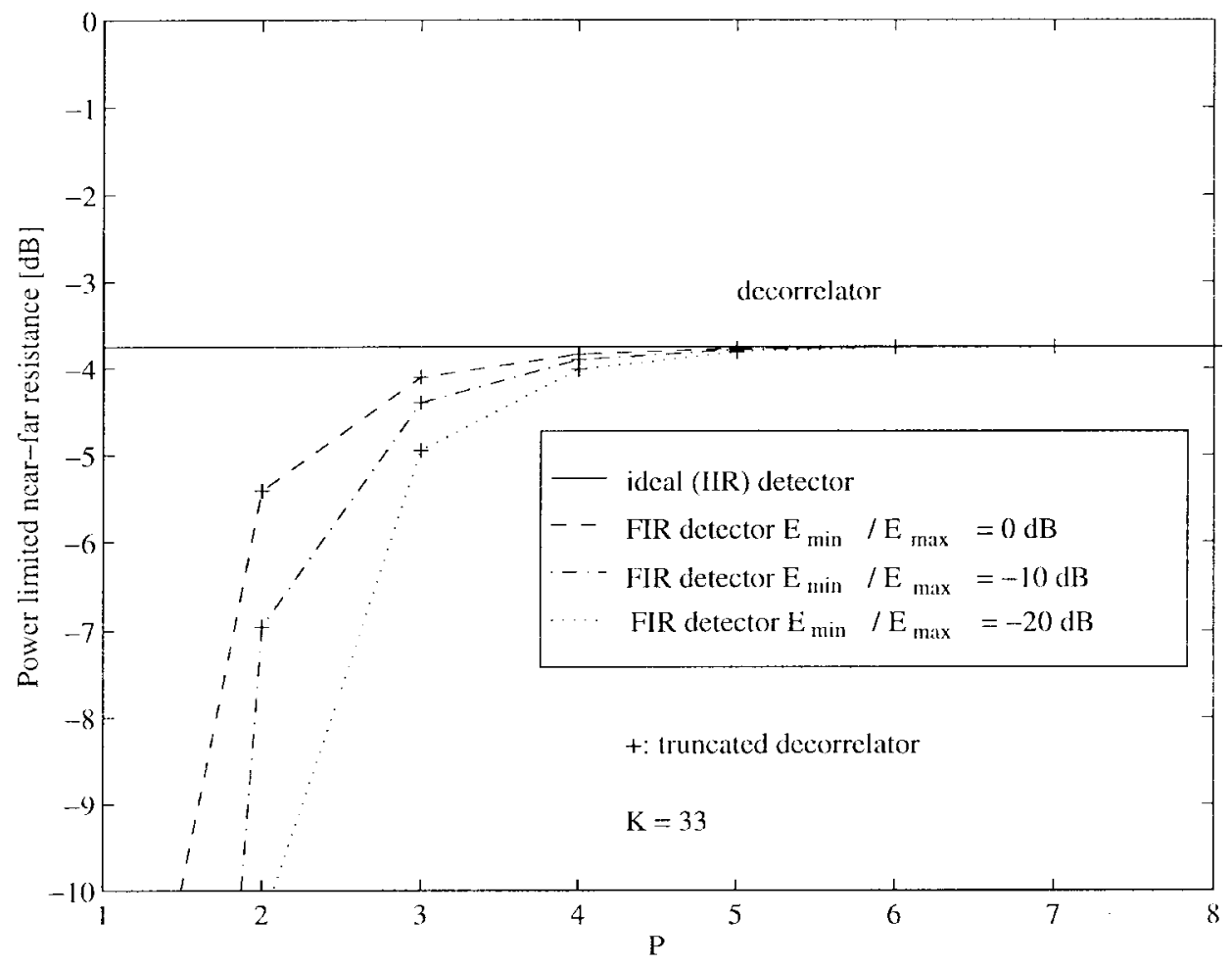

Fig. 4. Power-limited near-far resistances $[\mathrm{dB}]$ of truncated decorrelating detector as a function of parameter $P$ with time-variant signature waveforms.

approaches zero matrix as $N \rightarrow \infty$ since both $\mathbf{T}_{N-1, i}(N-1)$ and $\mathbf{T}_{N-1, j}(N-1) \rightarrow \mathbf{0}_{K}$ as $N \rightarrow \infty$. This guarantees the existence of a unique asymptotic limit for $\mathbf{T}_{i, j}(N-1)$. Thus, the uniqueness of the blocks $\mathbf{T}_{i, P+1}(N-1), i=-P, \cdots, P$ follows. Since the decorrelating detector $\mathcal{D}$ consists of the blocks $\mathbf{T}_{i, P+1}(N-1), i=-P, \cdots, P$ the uniqueness of the IIR decorrelating detector has been shown. The uniqueness of the LMMSE detector follows with exactly similar arguments. 


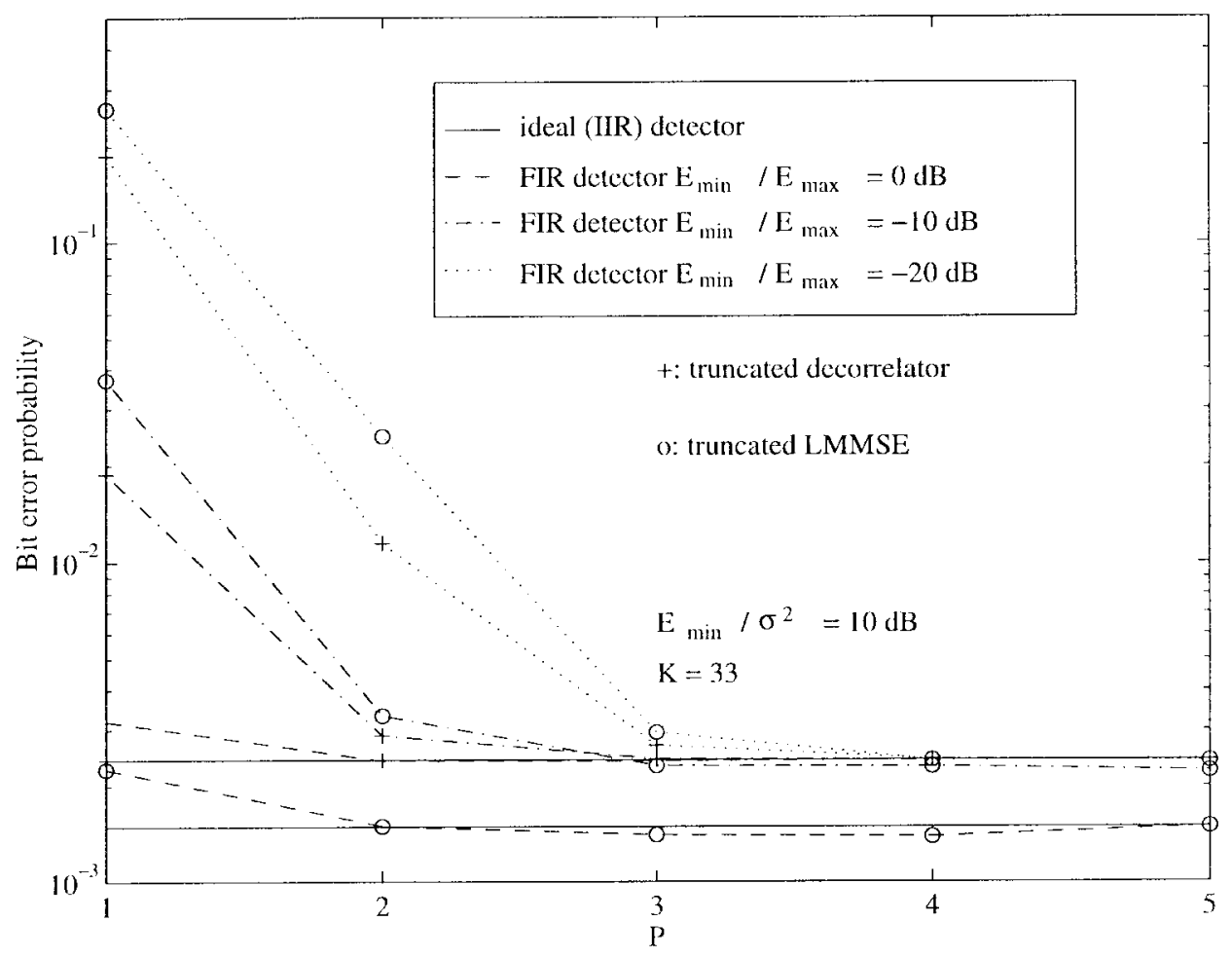

Fig. 5. Probabilities of bit error of truncated decorrelating detector as a function of parameter $P$ with time-variant signature waveforms.

The uniqueness of the noise-whitening detector follows easily from the uniqueness of the decorrelating detector by analyzing the Cholesky factor of $\mathcal{T}_{N}$ as $N \rightarrow \infty$.

Proof of Proposition 2: The result follows by manipulation of (13). By the definition of $\overline{\mathcal{R}}$, i.e., by $\overline{\mathcal{R}}=\left(\boldsymbol{\zeta}_{1}, \mathcal{R}, \boldsymbol{\zeta}_{2}\right) \in$ $\mathbb{R}^{N K \times(N+2) K}$, we can write

$$
\overline{\mathcal{R}}^{\top} \mathcal{R}^{-1} \overline{\mathcal{R}}=\left(\begin{array}{ccc}
\zeta_{1}^{\top} \mathcal{R}^{-1} \zeta_{1} & \zeta_{1}^{\top} & \boldsymbol{\zeta}_{1}^{\top} \mathcal{R}^{-1} \zeta_{2} \\
\boldsymbol{\zeta}_{2}^{\top} \mathcal{R}^{-1} \zeta_{1} & \zeta_{2}^{\top} & \zeta_{2}^{\top} \mathcal{R}^{-1} \zeta_{2}
\end{array}\right)
$$

Thus, except for the edges (the first and last $K$ columns and rows), the matrix $\overline{\mathcal{H}}=\left(\overline{\mathcal{R}}^{\top} \mathcal{R}^{-1} \overline{\mathcal{R}}+\sigma^{2} \overline{\mathcal{E}}^{-1}\right)$ is the same as $\mathcal{H}=\left(\boldsymbol{R}+\sigma^{2} \mathcal{E}^{-1}\right)$. If the assumptions of Proposition 2 are valid, $\mathbf{T}_{N, 1}(N) \rightarrow 0$ as $N \rightarrow \infty$, and $\boldsymbol{\zeta}_{1}^{\top} \mathcal{R}^{-1} \boldsymbol{\zeta}_{2}=$ $\mathbf{R}^{(n-P-1)}(1) \mathbf{T}_{N, 1}^{\top} \mathbf{R}^{\top(n+P+1)}(1) \rightarrow 0$ as $N \rightarrow \infty$. By utilizing the fact that the mathematical structures of the decorrelating and the LMMSE detectors are similar, it can be seen from (16) that the effect of the first and last diagonal blocks of $\overline{\mathcal{H}}$ on the middle block column of $\overline{\mathcal{H}}^{-1}$ goes to zero as $N \rightarrow \infty$. Thus, we have shown that

$$
\operatorname{mbc}\left\{\overline{\mathcal{H}}^{-1}\right\} \rightarrow \operatorname{mbc}\left\{\mathcal{H}^{-1}\right\}, \quad \text { as } N \rightarrow \infty \text {. }
$$

Recall that the optimal FIR LMMSE detector is the middle block column of $\mathcal{R}^{-1} \overline{\mathcal{R}} \overline{\mathcal{H}}^{-1}$ by (13). It is easy to verify by definitions that $\mathcal{R}^{-1} \overline{\mathcal{R}}=\left(\begin{array}{lll}\mathcal{R}^{-1} \boldsymbol{\zeta}_{1} & \boldsymbol{I}_{N K} & \mathcal{R}^{-1} \boldsymbol{\zeta}_{2}\end{array}\right)$. Matrix $\mathcal{R}^{-1} \overline{\mathcal{R}}$ is now identity, except for the first and last block columns. By the stability assumptions, the first and last blocks of the middle block column of $\overline{\mathcal{H}}^{-1}$ approach zero, so that the effect of the first and last blocks of matrix $\mathcal{R}^{-1} \overline{\mathcal{R}}$ vanishes asymptotically. Proposition 2 is now proved by the above and (28).

The Appendix is concluded by deriving conditions for (21) to be valid. First, note that by the definition of $\overline{\mathcal{R}}$, we have $\overline{\mathcal{R}} \overline{\mathcal{R}}^{\top}=\mathcal{R}^{2}+\zeta_{1} \zeta_{1}^{\top}+\zeta_{2} \zeta_{2}^{\top}$. The structure of the matrix $\overline{\mathcal{R}} \overline{\mathcal{R}}^{\top}$ is similar to that of $\mathcal{R}$. Furthermore, the matrix is the same as $\mathcal{R}^{2}$, except for the perturbations caused by $\zeta_{1} \zeta_{1}^{\top}$ and $\zeta_{2} \zeta_{2}^{\top}$ to the first and last diagonal blocks. Now, it is easy to understand that, under conditions similar to (19) being true, the middle block row (or column) of $\left(\overline{\mathcal{R}} \overline{\mathcal{R}}^{\top}\right)^{-1}$ approaches the middle block row (or column) of $\mathcal{R}^{-2}$. If, on the other hand, that is the case, it is easy to see from (12) that $\overline{\mathcal{D}}_{d} \rightarrow \mathcal{D}_{d}$ as $N \rightarrow \infty$ because the zero blocks of $\overline{\mathcal{R}}$ remove the effect of perturbations caused by $\zeta_{1} \zeta_{1}^{\top}$ and $\zeta_{2} \zeta_{2}^{\top}$ to the first and last diagonal blocks of $\overline{\mathcal{R}} \overline{\mathcal{R}}^{\top}$ as $N$ is sufficiently large.

\section{ACKNOWLEDGMENT}

The authors acknowledge M. Latva-aho of the University of Oulu and Dr. J. Lilleberg of Nokia Mobile Phones for many useful discussions.

$$
\begin{aligned}
&\left(\begin{array}{ll}
\mathbf{A} & \mathbf{B} \\
\mathbf{C} & \mathrm{D}
\end{array}\right)^{-1}=\left(\begin{array}{cc}
\left(\mathbf{A}-\mathbf{B D}^{-1} \mathbf{C}\right)^{-1} & -\left(\mathbf{A}-\mathbf{B D}^{-1} \mathbf{C}\right)^{-1} \mathbf{B D}^{-1} \\
-\left(\mathbf{D}-\mathbf{C A}^{-1} \mathbf{B}\right)^{-1} \mathbf{C A}^{-1} & \left(\mathbf{D}-\mathbf{C A}^{-1} \mathbf{B}\right)^{-1}
\end{array}\right) \\
&(\mathbf{A}+\mathbf{B C D})^{-1}=\mathbf{A}^{-1}-\mathbf{A}^{-1} \mathbf{B}\left(\mathbf{D A}^{-1} \mathbf{B}+\mathbf{C}^{-1}\right)^{-1} \mathbf{D A}^{-1}
\end{aligned}
$$




\section{REFERENCES}

[1] S. Verdu, "Minimum probability of error for asynchronous Gaussian multiple-access channels," IEEE Trans. Inform. Theory, vol. IT-32, pp. 85-96, Jan. 1986.

[2] _ _ "Multiuser detection," in Advances in Statistical Signal Processing, vol. 2. Greenwich, CT: JAI Press, 1993, pp. 369-409.

[3] R. Lupas and S. Verdu, "Near-far resistance of multiuser detectors in asynchronous channels," IEEE Trans. Commun., vol. 38, pp. 496-508, Apr. 1990.

[4] A. Klein and P. W. Baier, "Linear unbiased data estimation in mobile radio systems applying CDMA," IEEE J. Select. Areas Commun., vol. 11, pp. 1058-1066, Sept. 1993.

[5] Z. Xie, R. T. Short, and C. K. Rushforth, "A family of suboptimum detectors for coherent multiuser communications," IEEE J. Select. Areas Commun., vol. 8, pp. 683-690, May 1990.

[6] A. Duel-Hallen, "A family of multiuser decision-feedback detectors for asynchronous code-division multiple-access channels," IEEE Trans. Commun., vol. 43, pp. 421-434, Feb./Mar./Apr. 1995.

[7] F.-C. Zheng and S. K. Barton, "Near-far resistant detection of CDMA signals via isolation bit insertion," IEEE Trans. Commun., vol. 43, pp. 1313-1317, Feb./Mar./Apr. 1995.

[8] S. Moshavi, E. G. Kanterakis, and D. L. Schilling, "Multistage linear receivers for DS-CDMA systems," Int. J. Wireless Inform. Networks, vol. 3, no. 1, pp. 1-17, 1996.

[9] S. S. H. Wijayasuriya, G. H. Norton, and J. P. McGeehan, "A sliding window decorrelating receiver for multiuser DS-CDMA mobile radio networks," IEEE Trans. Veh. Technol., vol. 45, pp. 503-521, Aug. 1996.

[10] U. Madhow and M. L. Honig, "MMSE interference suppression for direct-sequence spread-spectrum CDMA," IEEE Trans. Commun., vol 42, pp. 3178-3188, Dec. 1994.

[11] P. B. Rapajic and B. S. Vucetic, "Adaptive receiver structures for asynchronous CDMA systems," IEEE J. Select. Areas Commun., vol. 12, pp. 685-697, May 1994.

[12] S. L. Miller, "An adaptive direct-sequence code-division multiple-access receiver for multiuser interference rejection," IEEE Trans. Commun., vol. 43, pp. 1746-1755, Feb./Mar./Apr. 1995.

[13] U. Mitra and H. V. Poor, "Adaptive receiver algorithms for near-far resistant CDMA," IEEE Trans. Commun., vol. 43, pp. 1713-1724, Feb./Mar./Apr. 1995

[14] M. L. Honig, U. Madhow, and S. Verdu, "Blind adaptive multiuser detection," IEEE Trans. Inform. Theory, vol. 41, pp. 944-960, July 1995.

[15] M. Rupf, F. Tarkoy, and J. L. Massey, "User-separating demodulation for code-division multiple-access systems," IEEE J. Select. Areas Commun., vol. 12, pp. 786-795, June 1994.

[16] Y. Bar-Ness and N. Sezgin, "Adaptive multiuser bootstrapped decorrelating CDMA detector for one-shot asynchronous unknown channels," in Proc. IEEE Int. Conf. Acoust., Speech, Signal Processing, Detroit, MI, May 1995, pp. 1733-1736.

[17] U. Madhow and M. L. Honig, "MMSE detection of direct-sequence CDMA signals: Performance analysis for random signature sequences," Preprint, 1994.

[18] L. Wei and L. K. Rasmussen, "A near ideal noise whitening filter for an asynchronous time varying CDMA system," IEEE Trans. Commun., vol. 44, pp. 1355-1361, Oct. 1996.

[19] M. K. Simon, J. K. Omura, R. A. Scholtz, and B. K. Levitt, Spread Spectrum Communications Handbook. New York: McGraw-Hill, 1994.

[20] S. Vembu and A. J. Viterbi, "Two different philosophies in CDMA-A comparison," in Proc. IEEE Veh. Technol. Conf., Atlanta, GA, Apr. 1996, pp. 869-873.

[21] Telecommunication Industry Association, Mobile Station-Base Station Compatibility Standard for Dual-Mode Wideband Spread Spectrum Cellular Systems, July 1993.

[22] M. J. Juntti, B. Aazhang, and J. O. Lilleberg, "Linear multiuser detection for R-CDMA," in Proc. Commun. Theory Mini-Conf., IEEE Global Telecommun. Conf., London, England, Nov. 1996, pp. 127-131.
[23] _ "Iterative implementation of linear multiuser detection for asynchronous CDMA systems," IEEE Trans. Commun., submitted for publication.

[24] J. G. Proakis, Digital Communications, 3rd ed. New York: McGrawHill, 1995.

[25] A. Duel-Hallen, "Decorrelating decision-feedback multiuser detector for synchronous code-division multiple-access channel," IEEE Trans. Commun., vol. 41, pp. 285-290, Feb. 1993.

[26] G. H. Golub and C. F. Van Loan, Matrix Computations, 2nd ed. Baltimore, MD: Johns Hopkins Univ. Press, 1989.

[27] S. Kay, Fundamentals of Statistical Signal Processing: Estimation The ory. Englewood Cliffs, NJ: Prentice-Hall, 1993.

[28] J. G. Proakis and D. G. Manolakis, Digital Signal Processing: Principles, Algorithms and Applications, 2nd ed. New York: Macmillan, 1992.

[29] G. Ludyk, "Stability of time-variant discrete-time systems," vol. 5 of Advances in Control Systems and Signal Processing. Braunschweig, Germany: Friedr. Vierweg \& Sohn, 1985.

[30] M. K. Varanasi and B. Aazhang, "Near-optimum detection in synchronous code-division multiple-access systems," IEEE Trans. Commun., vol. 39, pp. 725-736, May 1991.

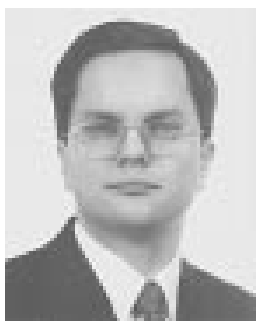

Markku J. Juntti (S'93) was born in Kemi, Finland, in 1969. He received the M.Sc. (Dipl. Eng.) degree in electrical engineering from the University of Oulu, Oulu, Finland in 1993. He is currently working towards the Ph.D. (Dr. Tech.) degree at the University of Oulu.

He has been a Research Scientist at the Telecommunication Laboratory, University of Oulu, since 1992. In the academic year 1994-1995, he was a Visiting Research Scientist at Rice University, Houston, TX. His research interests include communication theory and signal processing for wireless communication systems, with emphasis on multiuser receivers.

Behnaam Aazhang (S'82-M'85-SM'91) was born in Bandar Anzali, Iran, on December 7, 1957. He received the B.S. (with highest honors), M.S., and $\mathrm{Ph} . \mathrm{D}$. degrees in electrical and computer engineering from the University of Illinois at Urbana-Champaign in 1981, 1983, and 1986, respectively.

From 1981 to 1985 , he was a Research Assistant in the Coordinated Science Laboratory, University of Illinois. In August 1985, he joined the faculty of Rice University, Houston, TX, where he is now a Professor in the Department of Electrical and Computer Engineering. He has been a Visiting Professor at IBM Federal Systems Company, Houston, TX, the Institut für Kommunikationstechnik at Swiss Federal Institute of Technology (ETH), Zürich, Switzerland, the Telecommunications Laboratory at the University of Oulu, Oulu, Finland, and at the U.S. Air Force Phillips Laboratory, Albuquerque, NM. His research interests are in the areas of communication theory, information theory, and their applications, with emphasis on multiple-access communications, cellular mobile radio communications, optical communication networks, and the efficient simulation of stochastic systems.

Dr. Aazhang was the recipient of the Alcoa Foundation Award in 1993 , an NSF Engineering Initiation Award in 1987-1989, and an IBM Graduate Fellowship in 1984-1985, and is a member of Tau Beta Pi and Eta Kappa Nu. $\mathrm{He}$ is currently serving as the Editor for Spread Spectrum Networks of IEEE TRANSACTIONS ON COMMUNICATIONS and as the Treasurer of IEEE Information Theory Society. He has served as the Secretary of the Information Theory Society from 1990 to 1993, and as the Publications Chairman of the 1993 IEEE International Symposium on Information Theory, San Antonio, TX. 